\title{
Electrical Properties of Plastic Composite Materials with Rice-hull and Soy-hull Carbon Powders
}

\author{
K.Satou ${ }^{1}$, T.Takahashi ${ }^{2}$, H.Goto ${ }^{3}$, T.Kaneiwa ${ }^{4}$ and H.Iizuka ${ }^{1}$ \\ ${ }^{1}$ Yamagata University, Yonezawa Yamagata 992-8510, \\ ${ }^{2}$ Sanwa-yushi Co., Ltd., Higashine Yamagata 999-3716, \\ ${ }^{3}$ Nissin OilliO Group, Ltd., Chuo-ku Tokyo 104-8285 \\ ${ }^{4}$ Asahi Organic Chemicals Industry Co., Ltd., Niwa-gun Aichi 480-0105 \\ Fax: 81- 0238-26-3212, e-mail: dtk15044@dipfr.dip.yz.yamagata-u.ac.jp
}

\begin{abstract}
Rice-hull and soy-hull are agricultural by-products in Japan, and are required to be reused from a viewpoint of the recycling. The rice-hull and soy-hull carbon (RHC and SHC) powders are manufactured by carbonizing in nitrogen gas atmosphere at high temperature. The RHC and SHC carbon sheets are manufactured by wet papermaking process, in which the RHC and SHC powders, aramid fiber and LDPE (low density polyethylene) fiber are mixed in the water, and then drained the water to make the RHC and SHC carbon sheets. In this study, electrical conductivity, electromagnetic wave shielding and absorption properties of the RHC and SHC carbon sheets were discussed. Especially, the effects of the powder content, test-piece thickness, and the particle size of the RHC and SHC powders were measured. The result showed that the conductivity and the shielding effects were high in the SHC sheets with the large particle size. The absorption property was also high under certain high frequency areas for the RHC and SHC carbon sheets. Key words: Rice-hull, Soy-hull, Carbonization, Electrical conductivity, Electromagnetic wave shielding, Absorption
\end{abstract}

\section{INTRODUCTION}

Rice, that is the staple food of our country, is produced about 9 million tons per year, and the rice-hull of about 1.8 million tons is obtained as the by-product of the rice. Soybeans are imported about 4.2 million tons, and 3 million tons of those are used as raw materials for cooking oil such as the salad oil. The rice-hull and the soy-hull have been used as feed or manure. They are not effectively utilized, and are required to be reused as more profitable usage from a viewpoint of the recycling [1]. The agricultural by-products have a natural porous structure and contain some minerals ${ }^{[2]}$. The authors have performed some projects for the useful utilization of the rice-hull and soy-hull as an industrial carbon material ${ }^{[3,4]}$.

The rice-hull and soy-hull carbon (RHC and SHC) powders are manufactured by carbonizing those raw materials in a nitrogen gas atmosphere at high temperature. The RHC and SHC powders retain high electrical conductivity of the carbon. The present authors have developed the RHC and SHC powders for the industrial utilization. M.Shishido et al. have shown that the percolation phenomenon did not appear for the rubber composite materials with the RHC and SHC powders ${ }^{[5]}$. M.Kaneta et al. have revealed that the high electromagnetic shielding effect and the absorption property were obtained for the rubber composite materials with the RHC and SHC carbon powders ${ }^{[6]}$. Moreover, T.Suzuki et al. have proposed a new making process of the plastic composite material with the RHC and SHC carbon powders ${ }^{[7]}$.

In this study, electrical conductivity, electromagnetic wave shielding and absorption property of the RHC and SHC carbon sheets ware discussed. Especially, the effects of powder content, test-piece thickness, and particle size of the RHC and SHC powders were measured.

\section{EXPERIMENTAL PROCEDURE}

\subsection{Materials}

Figure 1 shows the manufacturing process of the RHC and SHC carbon sheets. The rice-hull was ground to break the hard surface layer. The ground rice-hull and the soy-hull ware carbonized at $1173 \mathrm{~K}$ in nitrogen gas atmosphere to make the RHC and SHC powders. Then the RHC and SHC powders ware pulverized to obtain the pulverized RHC and SHC powders. The non pulverized RHC and SHC powders or pulverized RHC and SHC powders were mixed in water with LDPE fiber and aramid fiber, and then drained the water to form the RHC and SHC carbon sheets. These sheets ware processed surface melting to make the RHC and SHC carbon sheets.

Table 1 lists the manufacturing conditions of the RHC and SHC carbon sheets. The diameter of the non pulverized RHC and SHC powder was about $350-500 \mu \mathrm{m}$. These powders ware pulverized. These median diameters ware about $60 \mu \mathrm{m}$.

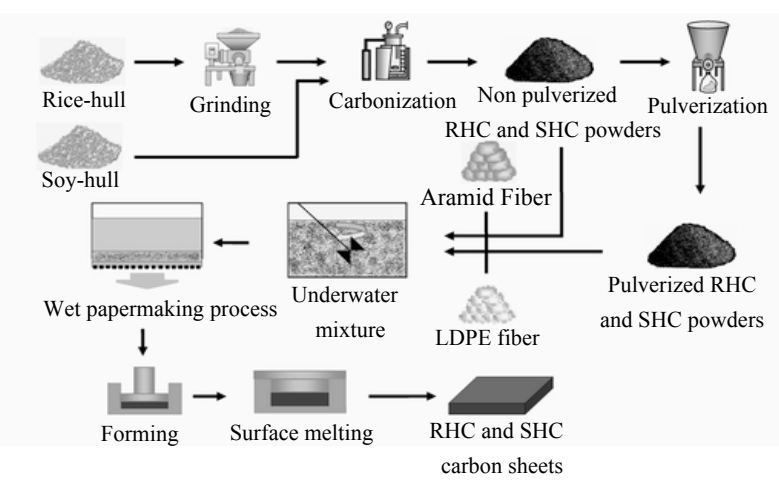

Fig.1 Manufacturing process of RHC and SHC carbon sheets. 
Table 1 Manufacturing conditions of RHC and SHC carbon sheets.

\begin{tabular}{|c|c|c|c|}
\hline PHC & Particle size $[\mu \mathrm{m}]$ & $\begin{array}{c}\text { Thickness, } \\
t[\mathrm{~mm}]\end{array}$ & $\begin{array}{c}\text { Powder } \\
\text { content } \\
{[\mathrm{wt} \%]}\end{array}$ \\
\hline SHC & $\begin{array}{c}60 \text { (pulverized), } \\
350-500 \\
\text { (non pulverized) }\end{array}$ & $3.0,4.5$ & $\begin{array}{c}50,60, \\
70,80\end{array}$ \\
\hline
\end{tabular}

\subsection{Experimental procedure}

Fig.2 shows the dimension of the test piece and the measuring parts for the resistivity test. The dimension of the test piece was $90 \times 90 \times t$ (thickness) $\mathrm{mm}^{3}$. The volume resistivity of the RHC and SHC sheets were measured using a resistivity meter.

Fig.3 shows the measuring system of the shielding effect, which was evaluated under 1-600 $\mathrm{MHz}$ by using the Advantest method. The dimension of the test-piece was $90 \times 90 \times t$ (thickness) $\mathrm{mm}^{3}$. The shielding effect (SE) was evaluated deducting the power of the transmitting wave from that of the incident wave.

The electromagnetic wave absorption property was evaluated under 2-8 $\mathrm{GHz}$ by using the Arch-testing method. Fig. 4 shows the measuring system of the Arch-testing method. The incident wave was applied from the transmitter to the test-piece, which dimension was $300 \times 300 \times t \mathrm{~mm}^{3}$. The electromagnetic wave absorption (reflectivity) was evaluated as the power loss between the incident wave and the reflective wave.

\section{EXPERIMENTAL RESULTS}

\subsection{Macrostructure}

Fig.5 (a) and (b) show the macrostructures of the non pulverized and pulverized RHC carbon sheets. The powders are entangled with the LDPE and aramid fibers. The dispersion of the powders was almost uniform. Similarly, the non pulverized SHC powders and pulverized SHC powders ware entwining the LDPE and aramid fibers, and the dispersion of the powders ware almost uniform.

\subsection{Electrical conductivity}

Fig.6 shows the effects of the test-piece thickness $(t)$, the powder content (pc), and the particle size on the volume resistivity for the RHC carbon sheets. The volume resistivity was about $10^{2}-10^{3} \cdot \mathrm{cm}$. Fig.7 shows the volume resistivity for the $\mathrm{SHC}$ carbon sheets. The volume resistivity was about $10^{1}-10^{2} \cdot \mathrm{cm}$.

The resistivity is slightly high in the RHC carbon sheet, because the rice hull contains about $20 \mathrm{wt} . \%$ of $\mathrm{Si}$ that is an insulating material ${ }^{[2]}$. The low resistivity in the non pulverized SHC carbon sheet attributes to the high continuity between the SHC powders.

\subsection{Electromagnetic wave shielding effect}

Fig.8 (a) and (b) show the effects of the test-piece thickness $(t)$, the powder content (pc), and the particle size on the electromagnetic wave shielding effect (SE) for the RHC carbon sheets. The shielding effect is higher than $20 \mathrm{~dB}$ under $10-100 \mathrm{MHz}$ radio frequency area for nearly half of the all sheets. The shielding effect was slightly increased with increasing the test-piece thickness. The effect of the powder content on the shielding was not large. The effect of the particle size on the shielding was large.

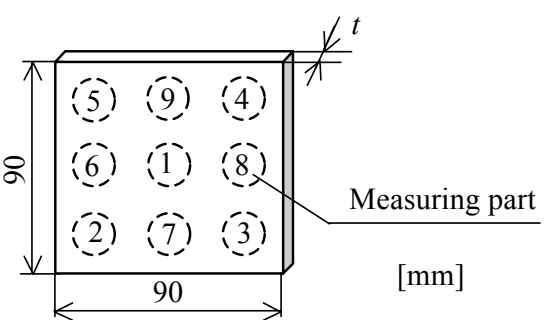

Fig.2 Dimension and measuring parts of test piece for resistivity.

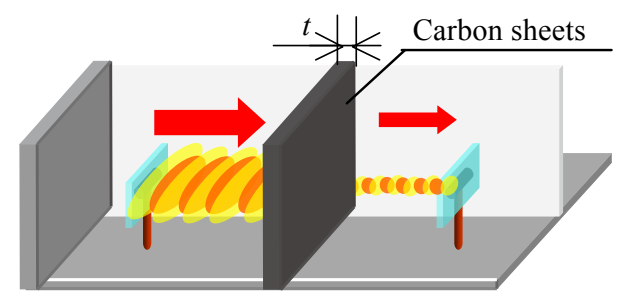

Fig. 3 Measuring system by using Advantest method for $1-600 \mathrm{MHz}$.

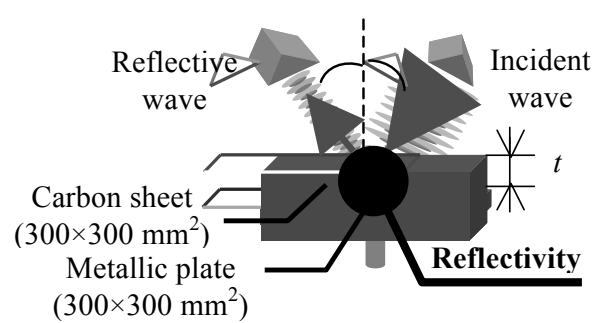

Fig.4 Measuring system by using Arch-testing method for $2-8 \mathrm{GHz}$.

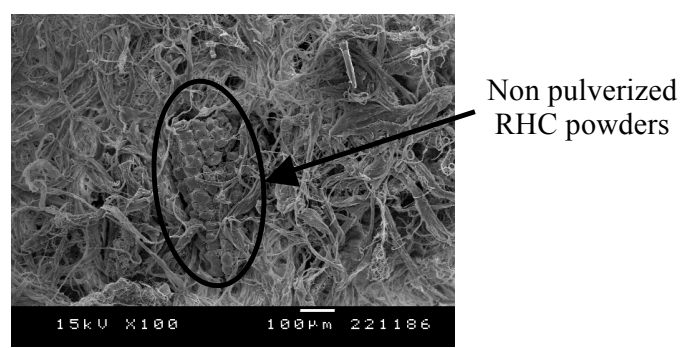

(a) Non pulverized

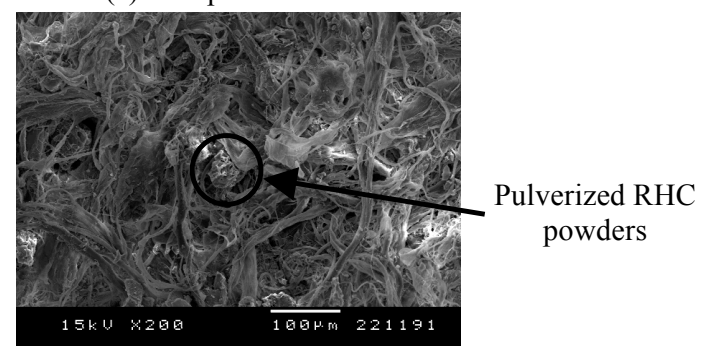

(b) Pulverized

Fig.5 Microstructure of RHC carbon sheets.

Fig.9 (a) and (b) show the effects of the test-piece thickness $(t)$, the powder content (pc), and the particle size on the electromagnetic wave shielding effect for the SHC carbon sheets. The shielding effect is higher than 20 $\mathrm{dB}$ under $10-100 \mathrm{MHz}$ radio frequency area. The shielding effect was slightly increased with increasing the test-piece thickness. The effect of the powder content on 


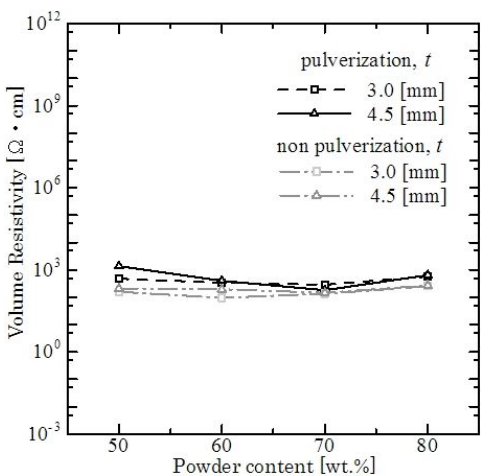

Fig.6 Volume resistivity for RHC carbon sheets.

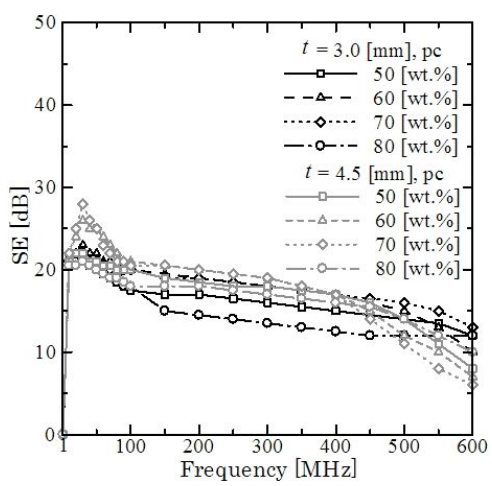

(a) Non pulverized

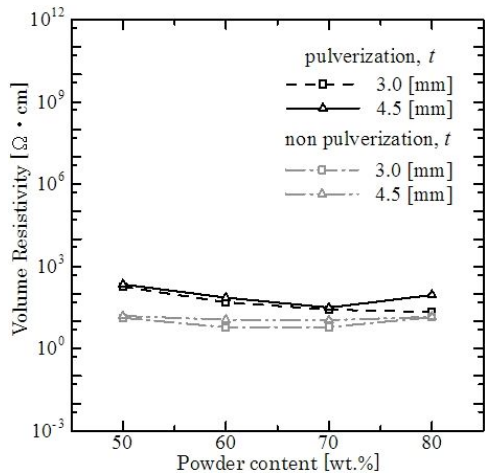

Fig.7 Volume resistivity for SHC carbon sheets.

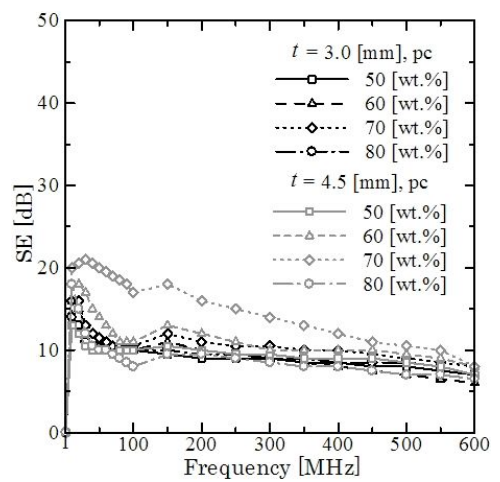

(b) Pulverized

Fig.8 Electromagnetic wave shielding effect for RHC carbon sheets.

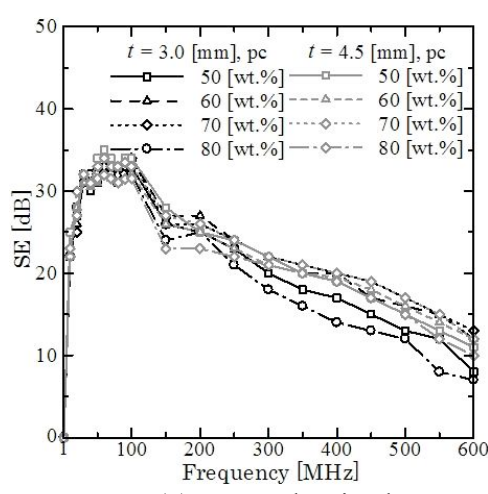

(a) Non pulverized

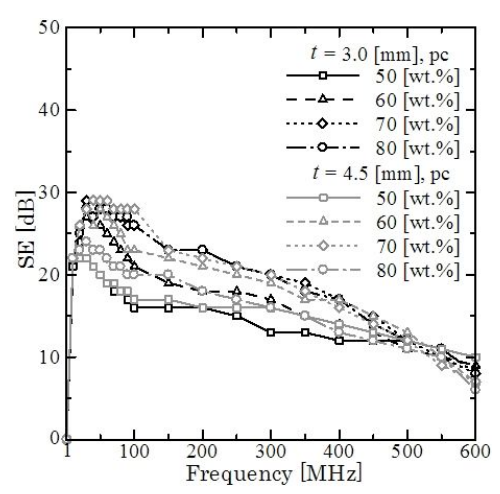

(b) Pulverized

Fig.9 Electromagnetic wave shielding effect for SHC carbon sheets.

the shielding was not large. The effect of the particle size on the shielding was large.

\subsection{Electromagnetic wave absorption property}

Fig.10 (a) and (b) show the effects of the test-piece thickness $(t)$, the powder content (pc), and the particle size on the electromagnetic wave absorption property (reflectivity) for the RHC carbon sheets. The absorption property is more than $20 \mathrm{~dB}$ in the radio frequency area with the pulverized sheets for nearly half of the all sheets. The effect of the test-piece thickness and the power content on the absorption was not large. The effect of the particle size on the absorption was large.

Fig.11 (a) and (b) show the effects of the test-piece thickness $(t)$, the powder content (pc), and the particle size on the electromagnetic wave absorption property for the SHC carbon sheets. The absorption is lower than 20 $\mathrm{dB}$ in the measurement range of radio frequency area for the almost sheets. The effect of the test-piece thickness and the power content on the absorption was not large. The effect of the particle size on the absorption was large.

\subsection{Consideration}

The rice-hull contains about $20 \mathrm{wt} . \%$ of $\mathrm{Si}^{[2]}$. Since $\mathrm{Si}$ is an insulating material, the electric conductivity was slightly lower in the RHC carbon sheet than in the SHC carbon sheet. Because the electromagnetic shielding effect is considered to be proportional to the conductivity, the shielding effect is higher in the SHC carbon sheet than in the RHC carbon sheet ${ }^{[8]}$. The main shielding effect is considered to be attributed to the reflecting effect on the test-piece surface ${ }^{[9]}$. The absorption property was higher in the RHC carbon sheet than in the SHC carbon sheet. Since the conductivity was high in the SHC carbon sheet, the electromagnetic wave was considered to be reflected at the surface of the SHC carbon sheet, and 


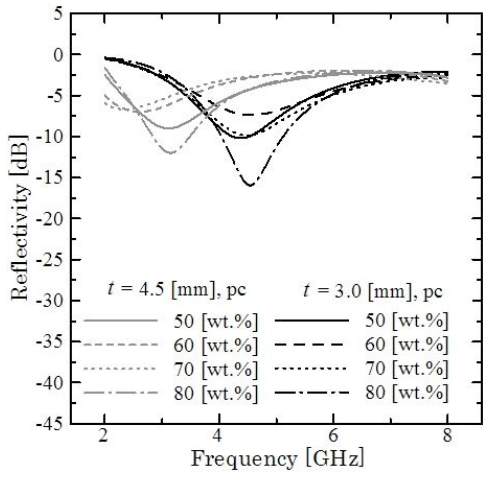

(a) Non pulverized

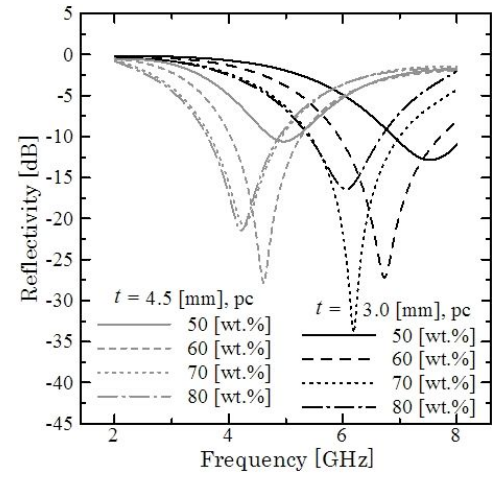

(b) Pulverized

Fig.10 Electromagnetic wave absorption properties for RHC carbon sheets.

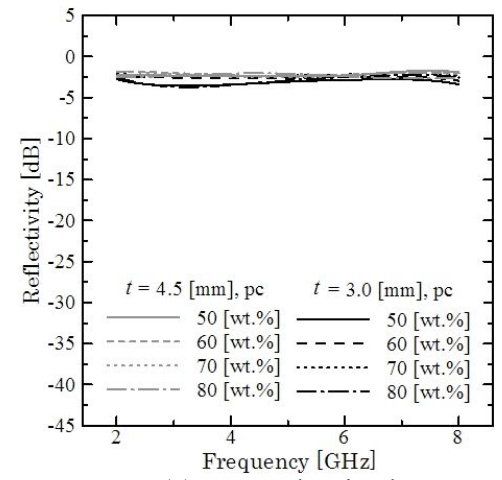

(a) Non pulverized

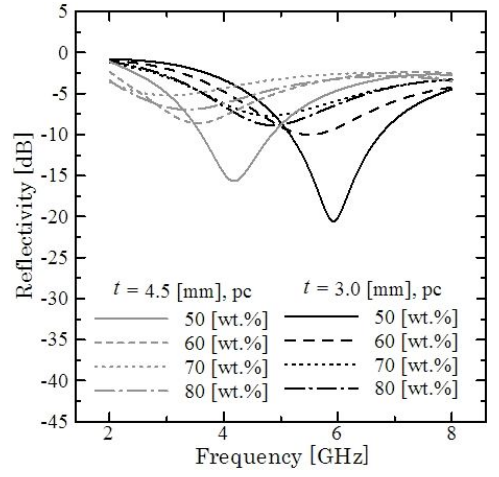

(b) Pulverized

Fig.11 Electromagnetic wave absorption properties for SHC carbon sheets.

therefore not absorbed at the inside of the SHC carbon sheet.

\section{CONCLUSION}

The electrical properties were measured for the plastic composite materials with rice-hull and soy-hull carbon powders. The summary of the obtained results are shown as follow.

(1) The volume resistivity was about $10^{1}-10^{3} \Omega \cdot \mathrm{cm}$ for the plastic composite materials with RHS and SHC carbon powders. The electrical conductivity was slightly lower in RHC carbon sheet than in SHC carbon sheet. The high conductivity was achieved in non pulverized SHC carbon sheet.

(2) The electromagnetic wave shielding effect was higher in SHC carbon sheet than in RHC carbon sheet. The high shielding effect was obtained in non pulverized SHC carbon sheet.

(3) The electromagnetic wave absorption property was higher in RHC carbon sheet than in SHC carbon sheet The high conductivity was considered to induce the high reflection at the test-piece surface, and not induce the high absorption at the inside of the sheet.

\section{REFERENCES}

[1] H.Iizuka, G.Kato, K.Hokkirigawa, S.Shikano and T.Takahashi, "Fracture Strength of a New Porous Carbon Material from Rice Bran", Proc. 3rd Int. Conf. on ECOMATERIALS, Tsukuba, Japan, 301-304 (1997)

[2] S.Chandrasekhar, K.G.Styanarayana, P.N.Pramada,
P.Raghavan, "Properties and Applications of Reactive Silica from Rice Husk - An Overview", J. Mater. Sci., 38, 3159-3168 (2003)

[3] H.Iizuka, G.Kato, K.Igarashi, S.Shikano and T.Takahashi, "Mechanical Properties of Porous Carbon Materials made from Rice Bran", J. Soc. Mater. Sci. Japan. 49, 625-630 (2000).

[4] M.Shishido, S.Kubo, T.Takahashi and H.Iizuka, "Mechanical Properties of Porous Carbon Materials made from Rice Hull", Trans. Mater. Res. Soc. Japan. 31, 993-996 (2006).

[5] M.Shishido, Y.Kurita, M.Ishikawa and H.Iizuka, "Improvement of Strength and Conductivity in Composite Materials with Rice-Hull Silica Carbon", Trans. Mater. Res. Soc. Japan., 32, 4, 1039-1042 (2007)

[6] M.Kaneta, H.Goto, T.Takahashi, H.Anzai and H.Iizuka, "Electromagnetic Wave Absorption in Rubber Composite Materials with Soy Hull Carbon Powder", Prec. 9th Int. Conf. on ECOMATERIALS, Kyoto, 125 (2009)

[7] T.Suzuki, T.Kaneiwa, T.Takahashi and H.Iizuka "Electric Properties of Composite Materials with Rice Hull Carbon", Prec. 6th Int. Works. on Green Composites, Sept. 8-10, 271-274(2010)

[8] K.Hatakeyama, "Manual of Hands-on Technique for Electromagnetic Shielding and Absorption (in Japanese)", (Mimatsu, Tokyo), 1-17(2006)

[9] K.Harada, "Technology for Electromagnetic Shielding and Absorption (in Japanese)", (Toray Research Center, Tokyo), 30-39(2008)

(Received July 5, 2011; Accepted December 27, 2011) 